\title{
LA NOUVELLE REPARTITION DES COMPETENCES ENTRE L'ETAT ET LA POLYNÉSIE FRANÇAISE
}

\author{
Jean Peres*
}

Les dispositions de la loi du 27 février 2004 conférant à la Polynésie française son nouveau statut de Pays d'outre mer, ont permis au gouvernement français de parfaire le processus d'autonomie déjà amorcé depuis maintenant plus de deux décennies. Cet article s'intéresse à la nouvelle répartition des compétences qui existe désormais entre l'Etat français et le gouvernement polynésien. L'auteur après avoir analysé les compétences qui relèvent encore du domaine des prérogatives de l'Etat français, propose aux lecteurs ce qui doit être maintenant considéré comme étant la vraie mesure institutionnelle de l'autonomie polynésienne actuelle. Il compare aussi les nouvelles dispositions constitutionnelles avec la loi statutaire du 12 avril 1996 pour mesurer le chemin parcouru (et ses limites) vers une plus large autonomie conférée au Territoire de la Polynésie française.

On 27 February 2004 the French government completed the process of giving French Polynesia the new status of Pays d'outre mer (overseas country). This article sets out the new division of powers between the French State government, and the French Polynesian government. Jean Peres analyses the areas that the French government has expressly retained in its sphere of competence, in order to assess the true extent of French Polynesian autonomy. He also compares the new arrangement with the law of 12 April 1996 to see how much the French Polynesian authority has been increased.

Les lois portant statut d'autonomie de la Polynésie française ${ }^{1}$ qui viennent d'être adoptées par le Parlement, validées pour l'essentiel par le Conseil Constitutionnel et publiées au Journal Officiel de

Conseiller spécial auprès du Président du Gouvernement de la Polynésie Française. Le texte ci-après représente le contenu de la conférence donnée par l'auteur, le 25 mars 2004, à l’Université de la Polynésie Française sous l'égide du service de la formation continue dans le cadre du programme «Savoir pour tous».

1 Loi organique n 2004-192 du 27 février 2004. Loi complétant la loi organique n 2004-193 du 27 février 2004. 
la Polynésie française du 12 mars 2004, procèdent, et c'est devenu une tradition depuis 1977, à une nouvelle répartition des compétences entre l'Etat et les institutions de la Polynésie française.

La première question qui se pose est de savoir s'il existe une frontière entre ce qui devrait rester de compétence de l'Etat et ce qui peut être délégué aux collectivités territoriales? Cette frontière, fluctuante d'une collectivité à l'autre et, pour une même collectivité, d'un statut à l'autre, n'était pas définie jusqu'à la dernière révision constitutionnelle et le Conseil Constitutionnel n'avait censuré jusqu'à présent que des dispositions qui faisaient que les garanties des libertés publiques pouvaient n'être pas les mêmes sur l'ensemble du territoire de la République. ${ }^{2}$

Un grand principe peut nous guider, celui de la subsidiarité, bien connu dans l'organisation de l'Union Européenne. Le deuxième alinéa de l'article 72 de la Constitution dispose que les collectivités territoriales ont vocation à prendre les décisions pour l'ensemble des compétences qui peuvent le mieux être mises en œuvre à leur échelon.

Mais ce principe généreux est aussitôt limité par le rappel de l'existence des compétences régaliennes, celles qui définissent l'existence d'un Etat souverain.

Le quatrième alinéa de l'article 74 de la Constitution issu de la révision de mars 2003, renvoyant sur ce point au quatrième alinéa de l'article 73, précise que le transfert de compétences de l'Etat ne peut porter sur:

- $\quad$ la nationalité,

- les droits civiques,

- $\quad$ les garanties des libertés publiques,

- l'état et la capacité des personnes,

- $\quad$ l'organisation de la justice,

- $\quad$ le droit pénal,

- la procédure pénale,

- $\quad$ la politique étrangère,

- $\quad$ la défense,

- $\quad$ la sécurité et l'ordre publics,

- la monnaie, le crédit et les changes,

- $\quad$ le droit électoral.

2 Comparer notamment décision n 96-373-DC du 9 avril 1996. 
Cette énumération précise et rigoureuse comporte toutefois deux atténuations.

La première sauvegarde les compétences qui étaient antérieurement exercées par les collectivités d'Outre-mer et qui ne sont pas remises en cause. Il en est ainsi, en ce qui concerne la Polynésie française, du droit pénal et de la politique étrangère. La seconde précise que les collectivités dotées de l'autonomie - ce qui est le cas de la Polynésie française - peuvent participer, sous le contrôle de l'Etat, à l'exercice des compétences conservées par l'Etat, mais dans le respect des garanties accordées sur l'ensemble du territoire national pour l'exercice des libertés publiques.

Dans cet exposé, je ne vous parlerai pas de la protection de l'emploi et de la protection du foncier, pas seulement parce que l'importance du sujet nécessiterait un exposé particulier, mais aussi parce que cette attribution de compétence à la Polynésie française découle directement de la Constitution, en contradiction avec les principes posés par l'article $1^{\text {er }}$ de cette Constitution qui assure l'égalité de tous devant la loi.

Le Conseil Constitutionnel en a aussitôt tiré la leçon en rappelant que les mesures qui seraient prises en faveur de la population doivent être strictement nécessaires à l'application des dispositions constitutionnelles dérogatoires.

Je vous propose d'examiner cette nouvelle répartition (I) en la comparant avec celle de la loi statutaire du 12 avril 1996. Par ailleurs, et même s'il ne s'agit pas d'un transfert de nouvelles compétences, la participation de la Polynésie française à l'exercice des compétences de l'Etat (II) contribuera à élargir les domaines dans lesquels peuvent intervenir, sous le contrôle de l'Etat, des normes fixées par les autorités polynésiennes.

\section{REPARTITION}

Si les principes généraux qui régissent cette répartition (A) ne sont guère différents de ceux qui prévalaient en 1996, au moins sur le fond, les compétences conservées par l'Etat (B) traduisent un net bouleversement et un changement d'orientation.

\section{A Principes Generaux}

Comme en 1996, la nouvelle loi statutaire consacre, dans son article 14, le principe selon lequel l'Etat exerce des compétences d'attribution ou d'exception alors que les institutions de la Polynésie française sont investies d'une compétence générale ou de principe. ${ }^{3}$

L'expression utilisée, pour définir les compétences de l'Etat, est la même dans les deux textes: «Les autorités de l'Etat sont compétentes dans les seules matières suivantes.» suivie d'une énumération de ces matières.

3 Voir également l'article 13 de la loi organique qui dispose que les autorités de la Polynésie française sont compétentes dans toutes les matières qui ne sont pas dévolues à l'Etat ou attribuées aux communes. 
La rédaction de 1996 était émaillée de restrictions, du genre «à l'exception de», «sous réserve de» ou «sans préjudice de» qui en rendaient la lecture malaisée.

Ces restrictions particulières ont quasiment disparu du texte de 2004 et ont été remplacées par un alinéa commun qui clôture l'article 14 et aux termes duquel:

Les compétences de l'Etat définies au présent article s'exercent sous réserve des pouvoirs conférés aux institutions de la Polynésie française par les dispositions de la section 2 du présent chapitre, et du titre

IV et de la participation de la Polynésie française aux compétences de l'Etat en application des dispositions de la section 3 du présent chapitre.

En d'autres termes, il faut se référer à la section qui traite des compétences particulières de la Polynésie française, au titre qui traite des compétences propres du président et du gouvernement par opposition à la compétence générale de l'assemblée - et enfin aux règles de la participation à l'exercice des compétences de l'Etat pour déterminer la réalité du domaine de compétence conservé par l'Etat.

A l'inverse, il a paru nécessaire d'encadrer les compétences nouvelles dévolues à la Polynésie française pour permettre à l'Etat d'exercer pleinement ses compétences dans le respect des sujétions imposées par la défense nationale.

C'est ainsi que l'Etat continuera à fixer les règles relatives:

- d'une part au droit du travail applicables aux salariés exerçant leur activité dans les établissements de l'Etat intéressant la défense nationale, en particulier la Direction des constructions navales;

- $\quad$ et d'autre part au transport, au stockage et à la livraison des produits pétroliers nécessaires à l'exercice par l'Etat de ses des missions de sécurité et de défense.

\section{B Competences de l'Etat}

Après avoir rappelé que tout ce qui n'est pas réservé à l'Etat relève de la compétence de la Polynésie française, je vous propose d'analyser cette réforme suivant les grands axes que j'ai regroupés ainsi:

- $\quad$ blocs de compétences;

- $\quad$ politique étrangère;

- $\quad$ administration communale;

- $\quad$ précisions et compléments;

- $\quad$ transferts vers l'Etat. 


\section{Transfert de blocs de compétence}

En matière de droit civil, l'Etat conserve la compétence pour tout ce qui touche:

- $\quad$ à la nationalité,

- à l'état et à la capacité des personnes, notamment les actes de l'état-civil, l'absence, le mariage, le divorce et la filiation,

- $\quad$ à l'autorité parentale,

- $\quad$ aux régimes matrimoniaux,

- $\quad$ aux successions et libéralités.

Il est permis de s'interroger sur l'autorité compétente en matière d'indivision.

En effet, une situation d'indivision peut naître, ce qui est généralement le cas d'une succession non partagée au moment du décès du propriétaire ou, plus exceptionnellement, de l'absence en cas de divorce de liquidation du régime matrimonial.

Les matières de succession et de régime matrimonial étant réservées à l'Etat, il semble logique d'y incorporer les dispositions du Code civil relatives à l'indivision et en excluant les autres dispositions de ce code qui traitent des conventions relatives à l'exercice en commun des droits indivis sur un bien.

A contrario, la Polynésie française devient compétente dans les matières suivantes:

- Définition et modifications de la propriété, y compris l'usufruit et les servitudes;

- Contrats et obligations conventionnelles, à l'exception des contrats de mariage, mais y compris les conventions relatives à l'exercice des droits indivis;

- Vente, échange ou louage de biens;

- Prêt, dépôt et séquestre;

- Mandat, cautionnement, nantissement, privilèges et hypothèques;

- Expropriation;

- Prescription et possession.

On peut avoir un doute en ce qui concerne le domicile, la tutelle, l'émancipation et la majorité qui ne sont pas expressément énumérés dans la rubrique intitulée état et capacité des personnes mais la liste n'est pas exhaustive puisqu'elle est précédée de l'adverbe «notamment» et mon opinion personnelle est que ces matières, traditionnellement rattachées à l'état et à la capacité des personnes, restent de la compétence de l'Etat. 
Je relève d'ailleurs au passage que ce «notamment» est en contradiction avec le principe affiché en tête de l'article 14 sur la limitation des matières relevant de la seule compétence de l'Etat. Il sera nécessaire, dans les cas litigieux, de solliciter l'avis du Conseil d'Etat, voire de saisir le Conseil Constitutionnel lorsqu'une loi de la République interviendra dans une matière relevant de la compétence de la Polynésie française.

Par contre, la procédure civile, bien qu'elle ne soit pas exclue du bloc du droit civil, reste bien une compétence de la Polynésie française ainsi que cela ressort des débats au Sénat au cours desquels Madame la ministre de l'outre-mer a rappelé le caractère limitatif des compétences de l'Etat.

Les autorités de la Polynésie française deviennent compétentes pour tout ce qui touche au droit du travail, y compris de la partie qui traite de l'inspection du travail, mais à l'exception:

- des tribunaux du travail qui relèvent de l'organisation judiciaire et

- des règles applicables aux salariés exerçant leur activité dans les établissements de l'Etat intéressant la défense nationale.

Le transfert à la Polynésie française du service d'Etat de l'Inspection du Travail sera subordonné à l'engagement du président de la Polynésie française d'accepter les obligations prévues par la convention internationale $n^{\circ} 81$ de l'organisation internationale du travail relative au statut des inspecteurs du travail. Au cours des débats au Sénat, il a été confirmé que le principe d'indépendance de ces agents n'était pas incompatible avec celui de l'autorité hiérarchique exercée dorénavant par le Gouvernement de la Polynésie française.

Enfin, comme pour tous les services d'Etat transférés, l'opération sera autorisée par décret fixant la date d'effet et les modalités du transfert, lesquelles seront finalement précisées par une convention passée entre le haut-commissaire et le président de la Polynésie française.

En matière de droit commercial, sous l'empire du statut de 1996, la Polynésie française n'était compétente que pour la partie réglementaire.

En vertu de la nouvelle loi, les principes fondamentaux des obligations commerciales, en d'autres termes les dispositions législatives du droit commercial, sont transférées à la Polynésie française. Ce transfert couvre également les règles relatives aux sociétés d'économie mixte, mais avec toutefois la restriction qui réserve à l'Etat le pouvoir d'édicter les règles relatives à l'administration communale. Autrement dit les dispositions de la loi n 83-597 du 7 juillet 1983 continueront de s'appliquer aux SEM associant la Polynésie française et les communes ou leurs groupements. ${ }^{4}$ Ces dispositions seront reprises dans la future ordonnance portant réforme du statut des communes.

4 A l'heure actuelle, aucune commune ne participe au capital d'une société d'économie mixte. 
Bien entendu, l'organisation et le fonctionnement des tribunaux de commerce continueront de relever de l'Etat au titre de l'organisation judiciaire.

\section{Politique étrangère}

La loi de 1996 utilisait l'expression de relations extérieures y compris en matière financière et commerciale, expression ambiguë et qui nécessitait des correctifs pour ne pas dépouiller complètement la Polynésie française des attributions qu'elle exerçait traditionnellement, à savoir les restrictions quantitatives à l'importation, les investissements étrangers, le régime douanier des marchandises à l'importation et à l'exportation, les règles de police vétérinaire et phytosanitaire et pour exclure de cette matière les pouvoirs propres du président du gouvernement dans le domaine des accords internationaux.

Le nouveau statut utilise l'expression de politique étrangère, conforme à la définition des pouvoirs régaliens donnée par la Constitution. La politique étrangère recouvre essentiellement l'activité diplomatique du gouvernement de la République et laisse une place assez large aux pouvoirs qui peuvent être confiés au président de la Polynésie française en matière de relations avec les Etats ou organismes internationaux du Pacifique et en matière de négociation d'accords internationaux, voire de conclusion pour certains d'entre eux ainsi que nous allons le voir.

En matière d'accords internationaux, rien n'est changé pour ceux qui interviennent dans des matières relevant de la compétence de l'Etat, à savoir que le président de la Polynésie française peut recevoir des autorités de la République les pouvoirs nécessaires pour négocier et signer au nom de la République. A titre d'exemple, le pouvoir donné au président de signer l'accord de Rarotonga sur l'interdiction des essais nucléaires dans le Pacifique. Lorsque ces accords interviennent dans des matières relevant de la compétence de la Polynésie française, l'habilitation préalable pour négocier a été supprimée. Le président de la Polynésie française doit soumettre le projet au Conseil des ministres et informer de son intention le Ministère des Affaires Etrangères qui peut s'opposer à cette négociation dans le mois qui suit sa saisine.

La signature de ces accords reste soumise à l'approbation par l'assemblée de la Polynésie française et au pouvoir délivré par les autorités de la République. Ils sont également soumis à approbation ou ratification dans les conditions prévues par la Constitution.

Les arrangements administratifs avec les administrations de tout Etat ou territoire du Pacifique sont négociés librement par le président de la Polynésie française. Ils recouvrent des accords de portée limitée ou de nature technique rendus nécessaires pour la mise en œuvre d'accords internationaux. Leur signature intervient après approbation par le conseil des ministres. ${ }^{5}$

5 A titre d'exemple, il est possible de citer les accords passés pour venir en aide aux victimes de catastrophes naturelles ou pour préciser les conditions d'exercice du contrôle sanitaire ou phytosanitaire. 
Il en est de même des conventions de coopération décentralisée, conclues avec des collectivités territoriales françaises ou étrangères. Par une réserve d'interprétation, le Conseil Constitutionnel a précisé que ces conventions devaient être approuvées par l'assemblée de la Polynésie française lorsqu'elles portent sur une matière relevant de la compétence de l'assemblée.

La Polynésie française pourra, avec l'accord des autorités de la République, devenir membre ou membre associé des organisations internationales du Pacifique ou observateur auprès de celles-ci.

Cette possibilité ouvre la porte, du côté français, à l'admission de la Polynésie française au Forum des Etats du Pacifique qui aura à se prononcer sur la candidature soit en qualité de membre associé, soit en qualité d'observateur.

Enfin, la Polynésie française pourra ouvrir des représentations auprès de tout Etat ou organisme international. Toutefois, il résulte tant des débats au Parlement que de la décision du Conseil Constitutionnel, que ces représentations ne sauraient avoir un caractère diplomatique, en d'autres termes qu'elles ne pourraient être des ambassades.

Elles pourront se consacrer, comme celles qui viennent d'être ouvertes en Chine, au développement des relations commerciales, à la promotion touristique de la Polynésie française ou au renforcement des liens culturels.

C'est le président de la Polynésie française qui négocie l'ouverture de ces représentations et qui nomme les représentants, après en avoir informé les autorités de la République et l'assemblée de la Polynésie française.

\section{Administration communale}

Le principe posé par l'article 72 de la Constitution et selon lequel aucune collectivité territoriale ne peut exercer de tutelle sur une autre n'est pas remis en cause. Et cependant la matière consacrée à l'administration communale a connu un véritable bouleversement.

Les règles relatives à l'administration, à l'organisation et aux compétences des communes, de leurs groupements et de leurs établissements publics ressortissent à la compétence de l'Etat. Toutefois, la Polynésie française peut déléguer aux communes les compétences qu'elle exerce en matière:

- d'aides et interventions économiques;

- d'aide sociale;

- d'urbanisme;

- de culture et patrimoine;

- de production et distribution d'électricité.

sous réserve de transférer à ces communes les moyens nécessaires à l'exercice de ces compétences. 
Après accord du conseil municipal, le maire peut recevoir délégation de compétence pour prendre les mesures individuelles d'application des lois du pays et des réglementations, ce qui peut concerner, par exemple, les règles de salubrité ou d'hygiène.

Dans les communes dotées d'un document d'urbanisme (PGA, POS), le gouvernement de la Polynésie française peut, sur la demande du conseil municipal, donner compétence au maire, agissant au nom de la commune, pour l'instruction et la délivrance des autorisations (permis de terrasser, de construire, de lotir) et des certificats d'urbanisme.

Tout ce qui touche à la coopération intercommunale (agglomérations, syndicats de communes) relève de la compétence exclusive de l'Etat.

Il en est de même du contrôle des actes des communes, de leurs groupements et de leurs établissements publics. Ce contrôle exercé actuellement «a priori» sera transformé en contrôle de légalité «a posteriori» après l'adoption de l'ordonnance étendant aux communes de Polynésie française les dispositions applicables aux communes de métropole depuis les lois de décentralisation de 1982 et 1983.

Les règles relatives au régime comptable et financier et au contrôle budgétaire des communes sont établies par l'Etat. Toutefois, dans ce domaine, la Polynésie française reçoit compétence pour définir les règles relatives aux marchés publics passés au nom des communes et aux délégations de service public consenties par les communes.

On peut citer également le pouvoir donné aux institutions de la Polynésie française de tracer le cadre d'une fiscalité spécifique aux communes. A l'intérieur de ce cadre, chaque conseil municipal arrête les taux et les modalités de perception des impôts et taxes institués. Il paraît évident que les principales difficultés seront la définition de l'assiette fiscale et l'importante disparité de potentiel fiscal existant entre la conurbation de Papeete et les autres communes. Il faut remarquer que la loi organique n'a pas saisi l'opportunité, offerte par le dernier alinéa de l'article 72-2 de la Constitution, de prévoir des dispositifs de péréquation destinés à favoriser l'égalité entre les collectivités, mais il est vrai que les règles du fonds intercommunal de péréquation permettent de satisfaire cette exigence. ${ }^{6}$

L'Etat est également compétent pour tout ce qui touche à la fonction publique communale et au domaine public communal. Le domaine initial d'une commune peut être constitué par décret à partir du domaine de la Polynésie française après avis conforme de l'assemblée. Il peut être étendu ultérieurement par délibération de l'assemblée sur avis conforme du conseil municipal.

6 Voir notamment le considérant 65 du Conseil Constitutionnel qui rappelle que la répartition ne doit pas méconnaître l'objectif d'égalité. 


\section{Précisions et compléments}

La rédaction de la loi de 1996, comme celle de 1984, a suscité, pour son exacte application en matière de répartition des compétences, de nombreuses demandes d'avis transmises au Conseil d'Etat. Il en a été de même lorsqu'au cours d'une instance contentieuse se posait la question d'une inexacte application de cette répartition des compétences.

L'objectif du législateur, en rédigeant l'article 14 de la loi organique, a été de supprimer ces zones d'incertitude. L'expérience nous dira s'il a réussi.

Mais je crois tout de même bon de signaler que les interrogations ont commencé avant même la promulgation de la loi statutaire.

La première s'est posée au moment de l'examen par l'Assemblée Nationale du projet de loi relatif à l'application du principe de laïcité dans les écoles, collèges et lycées publics. Après que le président de la Commission des lois, rapporteur du projet, ait conclu son rapport par une réaffirmation de l'application du principe de laïcité sur l'ensemble du territoire français, il précise que la loi ne s'appliquera pas à la Polynésie française car, en vertu de son statut d'autonomie, les établissements scolaires relèvent de la compétence des autorités de la Polynésie française.

Nous avons vu également qu'il pouvait y avoir un doute sur la question de l'indivision.

J'en viens à l'examen des principales précisions apportées par la loi statutaire.

1) Pour la desserte aérienne internationale, une ambiguïté de la loi de 1996 a été levée. Désormais l'Etat n'est compétent que pour les liaisons entre deux points du territoire de la République. En d'autres termes sur l'axe Papeete-Paris, l'Etat approuvera les programmes et les tarifs pour la liaison Papeete-Paris et la Polynésie française pour le tronçon Papeete-Los Angeles.

2) Dans le domaine monétaire, la loi précise que l'Etat est compétent en ce qui concerne les marchés financiers et pour la lutte contre la circulation illicite et le blanchiment des capitaux.

3) Les hydrocarbures liquides et gazeux ont été retirés de la liste des matières premières stratégiques. Cette liste résulte d'une ordonnance de 1959 du Président de la Communauté. Cette matière relève désormais de la compétence de la Polynésie française à l'exception des produits nécessaires aux missions de défense et de sécurité.

4) Dans le domaine maritime, la compétence de la Polynésie française est étendue à la sécurité des navires pour le transport des marchandises d'une jauge brute inférieure à 160 tonneaux, l'Etat restant compétent pour les navires jaugeant plus de 160 tonneaux et pour tous les navires destinés au transport des passagers. 
Il résulte de la décision du Conseil Constitutionnel que l'Etat reste compétent pour la sécurité publique et, en conséquence, pour la sécurité de la circulation et de la navigation dans les eaux territorials. ${ }^{7}$

Le conseil des ministres reçoit compétence pour la création d'un registre particulier d'immatriculation des navires, ce qui pourrait concerner les navires de croisière.

5) Pour la sécurité civile, l'Etat est responsable de la réquisition des moyens destinés aux secours pour faire face aux catastrophes, la loi de 1996 ne traitait que de la coordination de ces moyens.

6) En matière de justice, l'Etat a fait préciser sa compétence en ce qui concerne l'aide juridictionnelle que celle-ci intervienne en matière pénale ou en matière civile et administrative ${ }^{8}$ et pour la procédure administrative contentieuse.

7) L'enseignement supérieur devient l'enseignement universitaire afin de mieux affirmer la compétence de la Polynésie française sur les enseignements post-baccalauréat dispensés hors de l'Université (BTS, préparation aux grandes écoles, école normale, école des sages-femmes, arts et métiers).

8) Quant à la validité des diplômes d'enseignement, il est précisé que l'Etat est responsable de la collation et de la délivrance des grades, titres et diplômes nationaux, la loi de 1996 ne traitait que des diplômes sanctionnant les enseignements du second cycle du second degré.

9) Dans le domaine de la communication audiovisuelle, la Polynésie française pourra créer une ou plusieurs entreprises de production et de diffusions d'émissions audiovisuelles alors qu'en 1996, elle était limitée à une société de production et de diffusion d'émissions à caractère social, culturel et éducatif.

10) Dans la rubrique relative à l'entrée et au séjour des étrangers, le législateur a précisé que la compétence de l'Etat ne s'étendait pas aux règles relatives à l'accès au travail des étrangers, ce qui était déjà le cas en fait sous l'empire du statut de 1996.

11) L'Etat a tenu à préciser que les opérations de dénombrement de la population relevaient de sa seule compétence. En fait, les opérations de recensement ont toujours été réalisées par l'INSEE en étroite collaboration avec l'Institut polynésien de la statistique.

12) Autre précision en droit, mais qui ne change rien au fond: celle des règles de la fonction publique de l'Etat qui recouvrent l'ensemble de la fonction publique civile et militaire ainsi que celles des autres agents publics de l'Etat.

7 Les eaux territoriales comportent une bande de 12 miles nautiques (environ $20 \mathrm{~km}$ ) au-delà de la côte ou des lignes de base.

8 Seule la législation relative à l’aide juridictionnelle en matière pénale a été étendue à la Polynésie française. 
13) Après avoir confirmé la compétence de principe de la Polynésie française en ce qui concerne le régime douanier des marchandises à l'importation et à l'exportation, la loi précise que l'Etat reste compétent pour ce qui concerne les prohibitions à l'importation et à l'exportation qui relèvent de l'ordre public, et donc des règles de droit pénal et des engagements internationaux ratifiés par la France. A titre d'exemple, on peut citer le contrôle de la librairie, le commerce des stupéfiants et le contrôle des contrefaçons.

14) La loi précise, conformément à la Constitution, que l'Etat est compétent en matière de droit électoral, au titre des compétences régaliennes. Toutefois, la sécheresse de l'intitulé ne permet pas de répondre à toutes les questions, notamment celles qui recouvrent l'organisation des élections professionnelles ou consulaires, telle la Chambre de Commerce et d'Industrie. Il me paraît possible de considérer que la référence au droit électoral recouvre le contenu du code électoral, lequel exclut les élections consulaires et professionnelles.

\section{Transfert vers l'Etat}

1) A la demande des institutions de la Polynésie française, la compétence exercée jusque là en matière de réglementation relative à la liberté surveillée des mineurs, a été reprise par l'Etat au titre de sa responsabilité en matière de service public pénitentiaire auquel ont été ajoutés les services et établissements d'accueil des mineurs délinquants placés sur décision judiciaire.

Il était en effet devenu très difficile de coordonner les actions des services pénitentiaires ne relevant que de la responsabilité de l'Etat avec celles des services chargés de la surveillance des mineurs délinquants qui relevaient des affaires sociales.

2) Dans le statut de 1996 et depuis longtemps, l'Etat n'était compétent que pour «l'organisation législative de l'état-civil» ce qui laissait à la Polynésie française la responsabilité de la partie réglementaire, laquelle était notamment utilisée pour la forme de certains documents, tels les fiches d'état-civil. Le législateur et le Conseil Constitutionnel auraient pu faire application du principe de sauvegarde des compétences régaliennes exercées avant la réforme constitutionnelle de mars 2003. Il n'en a rien été.

La nouvelle loi ne comporte donc plus aucune restriction et tout ce qui touche aux actes d'étatcivil relève de la compétence de l'Etat sous réserve, nous le verrons dans la deuxième partie, de la possibilité de participation à l'exercice des compétences que l'Etat conserve.

\section{PARTICIPATION A L'EXERCICE DES COMPETENCES DE L'ETAT}

L'avant dernier alinéa de l'article 74 de la Constitution, dans sa rédaction de mars 2003, précise que la loi statutaire peut déterminer, pour les collectivités dotées de l'autonomie, les conditions dans lesquelles la collectivité peut participer, sous le contrôle de l'Etat, à l'exercice des compétences que l'Etat conserve, dans le respect des garanties accordées sur l'ensemble du territoire national pour l'exercice des libertés publiques. 
La nouvelle loi statutaire met ce principe révolutionnaire en œuvre en précisant d'une part la liste des domaines de compétence de l'Etat dans lesquelles la Polynésie française peut être habilitée à l'exercice de ces compétences (A) et d'autre part en détaillant, pour chaque catégorie d'acte, la procédure applicable (B).

\section{A Domaine de la Participation}

Il est précisé au préalable que la participation n'est pas un droit, mais une simple faculté soumise au bon vouloir de l'Etat. Il n'est pas inutile en effet de rappeler les propos de M Dominique Perben, garde des sceaux, à la tribune du Senat lors de l'examen de la loi constitutionnelle.

«S'agissant de compétences régaliennes, l'Etat conservera toujours le droit de s'opposer à un acte intervenu dans ce champ de compétence ou de le réformer, pour des motifs de légalité comme de pure opportunité.»

L'énumération qui suit ne reprend que la liste arrêtée à l'article 31 de la loi statutaire. On aurait pu, comme le Conseil Constitutionnel l'a fait dans ses commentaires, y ajouter la participation aux compétences internationales de l'Etat (voir ci-dessus IBb). J'ai préféré, pour des raisons de cohérence, en traiter au titre des compétences particulières de la Polynésie française.

\section{Etat et capacité des personnes}

Il convient de rapprocher l'expression de celle utilisée au $1^{\circ}$ de l'article 14 de la loi statutaire. Elle recouvre donc «notamment», les actes de l'état-civil, l'absence, le mariage, le divorce et la filiation.

La Polynésie française fonde de grands espoirs sur cette opportunité afin de:

- dynamiser la délivrance des actes d'état-civil nécessaires pour l'établissement des droits fonciers. Cette délivrance pourrait être assurée par un service central de l'administration polynésienne relevant de la direction des affaires foncières, elle suppose que soit réglée au préalable la question du registre d'état-civil qui servira de fondement à la délivrance de ces actes, elle suppose aussi que soit reconnue au responsable de ce service la qualité d'officier de l'état-civil. Aujourd'hui, cette qualité n'est reconnue en métropole qu'aux maires et aux adjoints et, à l'étranger, aux agents diplomatiques ou consulaires. En Polynésie française, cette qualité est également reconnue, en vertu d'arrêtés gubernatoriaux, au procureur de la République, autorité de tutelle en matière d'état-civil;

- adapter, pour tenir compte des particularités de la société polynésienne en matière de famille nourricière, les règles qui régissent la filiation adoptive; 
- assouplir les conditions de résidence pour les étrangers qui souhaitent contracter mariage en Polynésie française et qui ne peuvent respecter l'obligation de résidence minimum d'un mois. $^{9}$

\section{Autorité parentale}

Ce point n'appelle pas de commentaires particuliers et je n'ai pas connaissance de projets d'adaptation le concernant.

(a) Régimes matrimoniaux - successions et libéralités

La participation à l'exercice des compétences de l'Etat sur ces deux points me paraît intéressante pour apporter des solutions adaptées à la réalité polynésienne au problème de la sortie de l'indivision.

(b) Recherche et constatation des infractions

Jusqu'à aujourd'hui, les instances de la Polynésie française (assemblée et gouvernement) pouvaient assortir les infractions aux réglementations qu'elles édictaient de peines d'amende et même de peines d'emprisonnement, sous réserve pour ces dernières, d'une homologation législative.

Mais, faute de pouvoir rechercher les contrevenants, cette faculté restait trop souvent théorique, ce que n'a pas manqué de relever le Conseil Constitutionnel pour qui ${ }^{10}$ il est nécessaire de doter la Polynésie française du pouvoir d'édicter des règles de procédure pénale qui sont le prolongement nécessaire de l'exercice de ses compétences.

La nouvelle loi statutaire permet, dans le cadre d'une loi du pays, d'adopter des dispositions permettant aux fonctionnaires et agents assermentés des services de la Polynésie française de demander aux contrevenants, sous le contrôle de l'autorité judiciaire, de justifier de leur identité, de procéder à des consignations, des prélèvements d'échantillons, des saisies conservatoires, des retraits de la consommation, d'édicter des interdictions ou des prescriptions et de conduire les contrevenants devant un officier de police judiciaire. Ces agents peuvent même être habilités à effectuer des visites en présence d'un officier de police judiciaire requis. Toutefois, ils n'ont pas personnellement la qualité d'officier de police judiciaire. Agissant dans le cadre de la police administrative, ils doivent faire l'objet d'un agrément par le procureur de la République. Après avoir prêté serment devant le tribunal de première instance, ils sont commissionnés par le président de la Polynésie française. En cas de faute, l'agrément peut être retiré ou suspendu.

9 Aux termes de l'article 74 du Code civil, le mariage est célébré dans la commune où l'un des époux a son domicile ou sa résidence établie par une habitation continue depuis au moins un mois à la date de publication des bans.

10 Comparer considérant $n^{\circ} 52$. 
Les mêmes pouvoirs sont par ailleurs reconnus aux agents assermentés du port autonome et de la caisse de prévoyance sociale.

Bien entendu, les dispositions qui seront fixées par loi du pays ne peuvent s'écarter des limites et conditions fixées par la loi de l'Etat, et notamment le code de procédure pénale.

(c) Missions de police

Dans le domaine de la police judiciaire, la Polynésie française peut participer à l'exercice des missions de police incombant à l'Etat en matière de:

- $\quad$ surveillance et d'occupation du domaine public de la Polynésie française;

- police de la circulation routière;

- $\quad$ police de la circulation maritime dans les eaux intérieures;

Les contraventions à ces trois types de règlements doivent figurer sur une liste arrêtée par le Conseil des Ministres.

- missions de sécurité publique ou civile à la demande du haut-commissaire de la République. Les agents affectés à ces missions sont placés sous l'autorité opérationnelle des responsables de l'Etat. Ces missions peuvent être confiées à des fonctionnaires titulaires, des cadres territoriaux nommés par le président de la Polynésie française après agrément par le hautcommissaire et le procureur de la République et après prestation de serment devant le tribunal de première instance. Cet agrément peut être suspendu ou retiré pour faute par le haut-commissaire ou le procureur de la République.

Les dispositions de droit pénal en matière de jeux de hazard ne peuvent déroger aux règles législatives et réglementaires fixées par l'Etat en matière de contrôle (police des jeux) et de pénalités.

(d) Entrée et séjour des étrangers

La participation ne peut s'exercer en ce qui concerne l'exercice du droit d'asile, l'éloignement des étrangers et la circulation des citoyens de l'Union européenne, matières qui relèvent en tout ou en partie de conventions internationales.

(e) Communication audiovisuelle

- La réglementation qui sera prise par la Polynésie française dans ce domaine devra respecter les principes posés par la législation relative à la liberté de communication (loi n 86-1067 du 30 septembre 1986) en application du principe général relatif au respect des garanties accordées sur l'ensemble du territoire de la République. 
- En outre, tout projet ou proposition de loi du pays et tout projet de réglementation arrêté en Conseil des Ministres doit être soumis à l'avis préalable du Conseil supérieur de l'audiovisuel qui dispose d'un délai de trente jours pour rendre son avis.

(f) Services financiers des établissements postaux

Actuellement, seul un arrêté ancien d'un gouverneur des Etablissements français de l'Océanie réglemente le fonctionnement du centre de chèques postaux géré par l'Office des Postes et Télécommunications. Depuis la loi de 1977, qui a classé dans le domaine réservé à l'Etat tout ce qui concerne la banque et le crédit, aucune extension n'a pu être apportée aux services financiers de l'Office, les établissements de crédit ne voyant pas d'un bon œil une concurrence dans leur champ d'intervention. Il apparaît en particulier souhaitable que l'Office des Postes puisse offrir à sa clientèle, compte tenu du fait qu'il est présent sur toute l'étendue du territoire, des produits financiers tels que les contrats d'assurance-vie ou les plans d'épargne pour la retraite ou le logement.

\section{B Procédure}

La procédure utilisée est différente selon que la participation de la Polynésie française s'exerce dans le domaine législatif (a) ou réglementaire (b) étant précisé que dans ces domaines, la loi organique ne connaît que deux niveaux normatifs: ${ }^{11} \mathrm{La}$ loi du pays pour ce qui touche au domaine législatif et les arrêtés en Conseil des Ministres que ceux-ci soient pris pour l'application des lois du pays (pouvoir réglementaire dérivé) ou qu'ils interviennent dans le domaine du règlement (pouvoir réglementaire autonome). A aucun moment n'est envisagée l'hypothèse d'une délibération.

Mais, il existe toujours une ambiguïté sur la délimitation du domaine législatif. S'agit-il du domaine limitatif fixé par l'article 34 de la Constitution, hypothèse rejetée aujourd'hui par la doctrine et la jurisprudence du Conseil Constitutionnel ou plus largement du domaine d'intervention habituel de la loi métropolitaine? Dans le doute, nous retiendrons l'hypothèse la plus large, chaque fois que la Polynésie française souhaitera modifier une norme entrant dans le champ de la loi, c'est la procédure de loi du pays qui sera retenue.

\section{Domaine législatif}

- Ainsi que je viens de l'indiquer, tout acte intervenant dans le domaine législatif doit nécessairement revêtir la forme d'une loi du pays, ce qui signifie qu'il est soumis à la procédure instituée par les articles 139 à 146 qui prévoient notamment la consultation obligatoire du Haut-conseil de la Polynésie française et celle éventuelle du Conseil économique, social et culturel lorsque le sujet traite d'une matière à caractère économique ou social. Par ailleurs, cet acte est soumis aux dispositions des articles 176 à 180 qui traitent du contrôle juridictionnel spécifique des lois du pays, contrôle exercé par le Conseil d'Etat.

11 Comparer article LO 32 de la loi statutaire. 
- Le projet ou la proposition de loi du pays est transmis par le président de la Polynésie française - pour les projets - ou par le président de l'assemblée - pour les propositions - au ministre chargé de l'outre-mer qui en assure réception sans délai.

- Le ministre chargé de l'outre-mer et les autres ministres intéressés proposent au Premier Ministre, dans le délai de deux mois suivant la réception, un projet de décret tendant soit à l'approbation totale ou partielle de l'acte, soit au refus d'approbation.

- Le décret portant refus d'approbation doit être motivé.

- Le décret portant approbation est transmis, selon le cas, au président de la Polynésie française ou au président de l'assemblée. Le texte ne peut être adopté par l'assemblée que dans les mêmes termes que ceux du projet approuvé par l'Etat. Le droit d'amendement ne peut donc plus s'exercer et l'assemblée ne peut qu'approuver ou rejeter en bloc le projet présenté.

- La loi statutaire adoptée par le Parlement prévoyait que le décret d'approbation devenait caduc s'il n'était pas ratifié par la loi dans le délai de 18 mois suivant la signature du décret.

- Cette disposition posait bien évidemment la question de savoir ce qu'il adviendrait des dispositions de la loi du pays si la formalité substantielle de la ratification législative n'était pas respectée, soit que le projet de loi ne soit pas déposé, soit que le Parlement refuse de le voter, soit, hypothèse extrême, que la loi votée soit censurée par le Conseil Constitutionnel. Le décret devenant caduc, il en était de même de la loi du pays et des actes pris pour son application.

- La question n'a pas eu le temps d'être posée car le Conseil Constitutionnel (comparer considérant $n^{\circ}$ 45) a rappelé que la possibilité donnée à la Polynésie française d'édicter des normes dans un domaine qui demeurait dans les attributions de l'Etat ne pouvait résulter que de l'accord préalable de l'autorité de l'Etat qui exerce normalement cette compétence. Un texte entrant normalement dans le domaine législatif ne peut donc être approuvé que par le Parlement.

- Le Conseil a donc censuré l'expression «dans les dix huit mois de leur signature» et précisé (comparer considérant $n^{\circ} 49$ ) que la loi du pays ne pouvait entrer en vigueur tant que le décret d'approbation totale ou partielle n'a pas été ratifié par le Parlement.

- L'essentiel est donc sauvé, mais la procédure devient plus compliquée et plus longue, car si le Gouvernement de la République doit trancher dans les deux mois, il est bien évident qu'aucun délai minimum ne saurait être imposé au Parlement.

- Enfin, on peut s'interroger, pour ces lois du pays particulières qui auront été approuvées par décret, soumises à l'examen du Conseil d'Etat à travers la loi de ratification et peut-être déférées à la censure du Conseil Constitutionnel, sur l'utilité de conserver la procédure du 
contrôle juridictionnel spécifique qui permet à six représentants à l'assemblée ou à toute personne y ayant intérêt de déférer une loi du pays à l'examen du Conseil d'Etat.

- La controverse juridique n'est sans doute pas près de s'éteindre.

2 Domaine réglementaire

La loi organique distingue la procédure applicable aux arrêtés pris en Conseil des Ministres et celle applicable aux actes individuals:

(a) Arrêtés pris en Conseil des Ministres

- La procédure est la même qu'il s'agisse des arrêtés d'application des lois du pays décrites cidessus ou des arrêtés intervenant dans le domaine du règlement dans l'une des matières énumérées au II A.

- Le projet d'arrêté est transmis par le président de la Polynésie française au ministre chargé de l'outre-mer qui en accuse aussitôt réception.

- Comme pour les lois du pays, le ministre chargé de l'outre-mer et, éventuellement, les autres ministres intéressés proposent au Premier Ministre, dans le délai de deux mois, un projet de décret tendant soit à l'approbation totale ou partielle de l'acte, soit au refus d'approbation. Cette procédure du décret n'a pas été censurée par le Conseil Constitutionnel car l'approbation est donnée par l'autorité compétente en matière réglementaire. ${ }^{12}$

- Le refus d'approbation doit être motivé.

- L'acte approuvé par décret est soumis à l'examen du Conseil des Ministres qui doit l'adopter dans les mêmes termes, sans possibilité de modification ou le rejeter.

(b) Arrêtés individuels

- Les décisions individuelles prises en application des lois du pays ou des arrêtés en Conseil des Ministres doivent être transmises au haut-commissaire de la République, cette transmission subordonnant leur entrée en vigueur.

- Le haut-commissaire exerce un contrôle hiérarchique sur ces décisions. Le contrôle hiérarchique donne au haut-commissaire un pouvoir de réformation de l'acte sans qu'il soit nécessaire de le déférer au tribunal administratif.

- En matière d'entrée et de séjour des étrangers, le haut-commissaire peut s'opposer à la délivrance des titres de séjour par le Gouvernement de la Polynésie française et le Conseil Constitutionnel a pris soin de préciser que le contrôle hiérarchique s'exerce également sur

12 L’article 21 de la Constitution précise que le Premier Ministre exerce le pouvoir réglementaire. 
le refus de délivrance d'un titre de séjour (comparer considérant $\mathrm{n}^{\circ} 51$ ). Enfin, les modalités d'intervention du haut-commissaire en la matière doivent être fixées par décret.

- En matière de communication audiovisuelle, la loi statutaire précise que les décisions individuelles prises par les autorités de la Polynésie française, en application de la réglementation mise en place dans le cadre de la participation, peuvent être annulées ou réformées par le conseil supérieur de l'audiovisuel à la demande du haut-commissaire de la République ou de toute personne justifiant d'un intérêt pour agir.

Pour en terminer avec la participation, il convient de préciser que la loi organique a prévu que les lois du pays et les arrêtés en conseil des Ministres, intervenant dans le domaine de la participation, peuvent être à tout moment modifiés par une loi, une ordonnance ou un décret comportant une mention expresse d'application en Polynésie française. Cette possibilité illustre la déclaration de Monsieur Perben sur le droit absolu que l'Etat conserve sur la mise en œuvre de ces matières régaliennes.

On voit ainsi combien cette participation - ou association - est étroitement encadrée et contrôlée par l'Etat, condition qui a permis au Conseil Constitutionnel de valider ces procédures.

En conclusion, peut-on déduire de cette nouvelle répartition des compétences entre l'Etat et la Polynésie française qu'elle constitue un progrès substantiel pour l'exercice d'une véritable autonomie, gage de stabilité et de développement?

Indépendamment des précisions juridiques apportées par le texte à la définition des compétences de l'Etat, ce qui devrait, sinon supprimer, au moins réduire les contentieux qui ont émaillé la période 1996-2004, le transfert à la Polynésie française du bloc de compétence relatif aux obligations civiles et commerciales devrait faire disparaître l'écran horizontal qui limitait sérieusement les pouvoirs de l'assemblée.

Il n'était pas rare en effet qu'une réglementation, prise dans une matière qui relevait incontestablement de la compétence de la Polynésie française, telle par exemple la procédure civile, soit partiellement censurée parce que certaines de ses dispositions touchaient au droit civil. Cet obstacle a maintenant disparu et la sécurité juridique de nos réglementations s'en trouvera renforcée.

La loi organique, ${ }^{13}$ toujours sur le fondement de l'article 74 de la Constitution, a également institué une procédure originale de sauvegarde des compétences des autorités de la Polynésie française. Le Conseil Constitutionnel, saisi en ce sens, peut ainsi constater qu'une loi postérieure à la promulgation de la loi statutaire est intervenue dans une matière ressortissant à la compétence de la Polynésie française. Si tel est le cas, la loi intruse peut être modifiée par l'assemblée de la Polynésie française. 
Au terme de cet exposé, il est permis de constater que le législateur, poussé en cela par les propositions du Gouvernement de la Polynésie française et les amendements de ses parlementaires, a su aller jusqu'au bout des possibilités nouvelles ouvertes par la réforme constitutionnelle de mars 2003.

Il me paraît difficile aujourd'hui d'aller plus loin dans le domaine des compétences dévolues à la Polynésie française, mais il n'est pas défendu de se poser la question de savoir si on peut aller plus loin dans le domaine élargi de l'autonomie sans risquer de franchir la ligne jaune qui sépare cette autonomie au sein de la République de l'indépendance.

Il reste toutefois un domaine à explorer, sous réserve d'une nouvelle modification de la Constitution, c'est celui de la valeur des actes dénommés lois du pays. Lorsque l'on voit la lourdeur de la procédure, il faudra entre six mois et un an pour faire adopter une loi du pays, et le luxe de précautions prises pour que ces actes puissent être validés, on ne peut que regretter d'avoir tous les inconvénients d'une loi sans en avoir tous les avantages. 